\title{
CHANGE DETECTION FOR UPDATES OF VECTOR DATABASE THROUGH REGION- BASED CLASSIFICATION OF VHR SATELLITE DATA
}

\author{
Carleer Alexandre ${ }^{\mathrm{a}, *}$, Wolff Eléonore ${ }^{\mathrm{a}}$

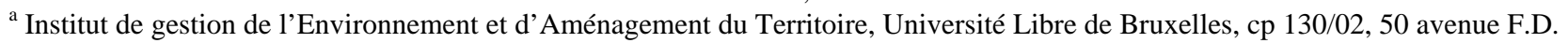 \\ Roosevelt, 1050 Brussels, Belgium, acarleer,ewolff(@ulb.ac.be)
}

KEY WORDS: Change detection, Database updating, Very high spatial resolution satellite images, Object based classification, PLEIADES-HR data

\begin{abstract}
:
Until now, interpretation of aerial photographs is a standard tool for monitoring land cover change where fine spatial resolutions are required and this task is expensive and time-consuming. Though, from a spaceborne perspective, the VHR satellite data are, since 1999, capable to meet the mapping and monitoring needs of municipal and regional planning agencies. Indeed, these data from the sensors Ikonos, QuickBird, OrbView-3, and in near future, the Pléiades-HR French sensors, have spatial resolution lower than $5 \mathrm{~m}$ in multispectral mode and lower than $1 \mathrm{~m}$ in panchromatic mode. These new sources of data combine the advantages of satellite data (synoptic view, digital format suitable for computer processing, quantitative land surface information at large spatial coverage and at frequent temporal intervals ...) with the very high spatial resolution.

In spite of these advantages, the use of VHR satellite data involves some problems in traditional per-pixel classification often used in change detection techniques. There are still two occurring classification problems that can strongly deteriorate the result of a perpixel classification of the VHR satellite data: spectral variability and poor spectral resolution. A solution to overcome these problems is the region-based classification that can be integrated in the common change detection techniques. The segmentation, before classification, produces regions which are more homogeneous in themselves than with nearby regions and represent discrete objects or areas in the image. Each image region then becomes a unit analysis and makes it possible to avoid much of the structural clutter. Image segmentation provides a logical transition from the units of pixels to larger units in maps more relevant to detect the changes in these.
\end{abstract}

In this context, this research project suggests to use region based classification of VHR satellite data in the change detection processe for updates of vector database.

\section{INTRODUCTION}

Up-to-date knowledge of land cover is an important tool for the various planning authorities with responsibilities for the management of territory (Marçal et al., 2005). The geospatial objects are changing over time and the land cover information (vector geospatial database) has to be up-date periodically. In general, change detection in a vector geospatial database involves the application of multi-temporal datasets to quantitatively analyse the changes. Because of the advantages of repetitive data acquisition, satellite remotely sensed data, such as Satellite Pour l'Observation de la Terre (SPOT), Thematic Mapper (TM), and Advanced Very High Resolution Radiometer (AVHRR), have become major data sources from local to global scales for different change detection applications during the past decades (Lu et al., 2003). However, planners and land managers require VHR data to address land cover problems at higher order thematic levels where spatial resolutions of $5 \mathrm{~m}$ or lower are required (Rogan and Chen, 2004).

The vector geospatial databases at a local scale in Belgium (e.g. National Geographic Institute 1/10000 topographic maps) were carried out by digital cartographic production process based on aerial photographs, and the update of these databases is now at the agenda.

Until now, interpretation of aerial photographs is a standard tool for monitoring land cover change where fine spatial resolutions are required (Loveland et al., 2002, Weis et al., 2005) and this task is expensive and time-consuming.

Though, from a spaceborne perspective, the VHR satellite data are, since 1999, capable to meet the mapping and monitoring needs of municipal and regional planning agencies (Treitz P., J. Rogan, 2004). Indeed, these data from the sensors Ikonos,
QuickBird, OrbView-3, and in near future, the Pléiades-HR French sensors, have spatial resolution lower than $5 \mathrm{~m}$ in multispectral mode and lower than $1 \mathrm{~m}$ in panchromatic mode. These new sources of data combine the advantages of satellite data (synoptic view, digital format suitable for computer processing, quantitative land surface information at large spatial coverage and at frequent temporal intervals ...) with the very high spatial resolution (Prenzel, 2004).

\section{CHANGE DETECTION TECHNIQUES}

The change detection techniques can be divided in two main types according to the data used to detect the changes: the image-database change detection and the image-image change detection.

In the first type the interpreted image is directly compared with the vector geospatial database.

Knudsen and Olsen (2003) detected the changes between a vector geospatial database and scanned aerial photographs, focusing on 'building' object class. They spectrally classified the scanned aerial photographs and overlaid the classification with the vector database in order to detect the new building.

Walter (2004) used a region-based classification for change detection. In this method, the objects, coming from the vector geospatial database that must be update, are spectrally classified and compared with the original database to detect the changes. This method is effective if a major change occurs in the original regions. Indeed, a change in the landscape can only be detected if it affects a large part of an object because the object-based classification uses the existing object geometry. If, for example, a building is built in a large forest area, this method fails to detect this new building. 
In the second change detection type, Dai and Khorram (1999) characterize the image-image change detection techniques by their functionalities and the data transformation procedures. They define two broad categories:

- The techniques where only changes and non-changes are detected and no categorical change information can be directly provided (for example, image differencing, image rationing and image regression); and

- The techniques where complete categorical changes are extracted (for example, post-classification comparison and direct multidate classification).

In the first category, changed and non-changed areas are separated by a preset threshold when comparing the spectral reflectance values of multitemporal satellite images (Dai and Khorram, 1999). Then, the amount of change is a function of the preset threshold, determined by experiments in the tails of the histogram representing change information (Lu et al., 2003), that constitute a critical step of the change/no-change detection techniques. Moreover, these techniques involve often radiometric calibration between dates.

The simple detection of change is rarely sufficient in itself: information is generally required on the initial and final land cover types - the 'from-to' analysis where complete categorical changes are extracted (Fuller et al., 2003). The major advantage of the second category is the capability of providing a matrix of change information (transition detection matrix) and reducing external impact from atmospheric and environmental differences between the multi-temporal images (Lu et al., 2003). Post-classification (delta classification) comparison is a common approach used for change detection (Lu et al., 2003). The principal advantage of delta classification lies in the fact that the two dates of imagery are separately classified, thereby minimizing the problem of radiometric calibration between dates (Coppin et al., 2004). However, the accuracy of the post-classification comparison is totally dependent on the accuracy of the initial classifications. The final accuracy very closely resembles that resulting from the multiplication of the accuracies of each individual classification (Petit and Lambin, 2001, Coppin et al., 2004).

In practice, an analyst often selects several methods to implement change detection in a study area, then compare and identify the best results through accuracy assessment (Lu et al., 2003) but among all 'from-to' change detection techniques, a supervised digital classification is used and this type of classification is generally applied on a per-pixel basis.

\section{REGION-BASED CLASSIFICATION}

In this framework, there are still two occurring classification problems that can strongly deteriorate the result of a per-pixel classification of the VHR satellite data (Irons et al., 1985, Smith and Fuller, 2001, De Wit and Clevers, 2004): spectral variability and poor spectral resolution. The spectral variability tends to reduce per-pixel classification accuracies and the resulting per-pixel classification will have a speckled appearance (salt-and-pepper effect) (Smith and Fuller, 2001). Besides the problem of increased variability, the VHR data have a relatively poor spectral resolution. Generally, there is a trade-off between the spatial resolution and the spectral resolution (Alpin et al., 1997, Key et al., 2001). This poor spectral resolution of the VHR satellite data can lead to problems in land cover interpretation (Herold et al., 2003).

Various solutions were developed to overcome these problems. For example, one possibility is proposed by Cushnie (1987) and Marceau et al. (1990) and consists of applying mathematical transformation to the original data to remove the excess spectral detail which is considered as noise (Cushnie, 1987). Some transformations are applied to the whole feature space (Principal Component Analysis ...), while others are applied to individual bands through the process of spatial linear filtering. (e.g. mean-filter). This solution represents a reductionist approach, in the sense that they attempt to solve the problem of higher spectral confusion by eliminating part of the information that is present in the images.

Another solution is the region-based classification that can be integrated in the common 'from-to' change detection techniques. The segmentation, before classification, produces regions which are more homogeneous in themselves than with nearby regions and represent discrete objects or areas in the image. Each image region then becomes a unit analysis and makes it possible to avoid much of the structural clutter. Image segmentation provides a logical transition from the units of pixels to larger units in maps (Ryherd and Woodcock, 1996) more relevant to detect the changes in these.

Moreover, the image segmentation provides a good mean for the integration of land cover information from remotely sensed data in GIS, where the geospatial database are usually stored (Marçal et al., 2005) and provides to the classification process information that could not be derived from single pixel such as context and shape features. These are very important factor to photo-interpretation and image understanding. The use of additional features could allow to compensate for the poor spectral resolution of VHR satellite data (Guindon, 2000, Herold et al., 2002) and to increase the classification accuracy for spectrally heterogeneous classes (Lillesand and Kiefer, 1994).

In this context, the integration of the region-based classification in change detection techniques in order to detect the changes in local/regional geospatial vector database with VHR satellite data could be relevant.

\section{STUDY ZONE AND DATA}

The study area is situated in the southeast of Belgium, near the frontier with Luxembourg. The image data are panchromatic and XS QuickBird images acquired on 12 May 2004 with a spatial resolution of $0.6 \mathrm{~m}$ in the panchromatic band and $2.4 \mathrm{~m}$ in the multispectral bands. The study area covers $59 \mathrm{~km}^{2}$ but for this study a $900 \mathrm{mx} 900 \mathrm{~m}$ study zone was used.

This study zone was extracted from the orgthorectified QuickBird image and from the TOP10V-GIS IGN vector database. The TOP10v-GIS database is the vector Belgian National Geographical Institute 1/10000 topographic map. This land use/cover database is constituted of 93 classes. Then, a generalization is essential.

With the satellite image and the vector database, a class transition reference was made. This reference allows to assess the change detection.

\section{METHOD}

The image - database change detection was chosen for this study in order to avoid radiometric correction, errors accumulation with the post-classification comparison and to use a priori knowledge from the vector database.

In order to directly integrate the data, the database can be used in the segmentation process and constrains this. Then the database is used as basis of the image segmentation. Each region of the database is segmented in order to detect the 
different objects that compose them. That ensures the integration and the matching at the same time.

These objects are classified and compared with the database classes in order to detect the changes. Another advantage of this method is to keep all database information and to do the generalization at different step of the process on the database and on the segmentation at the same time.

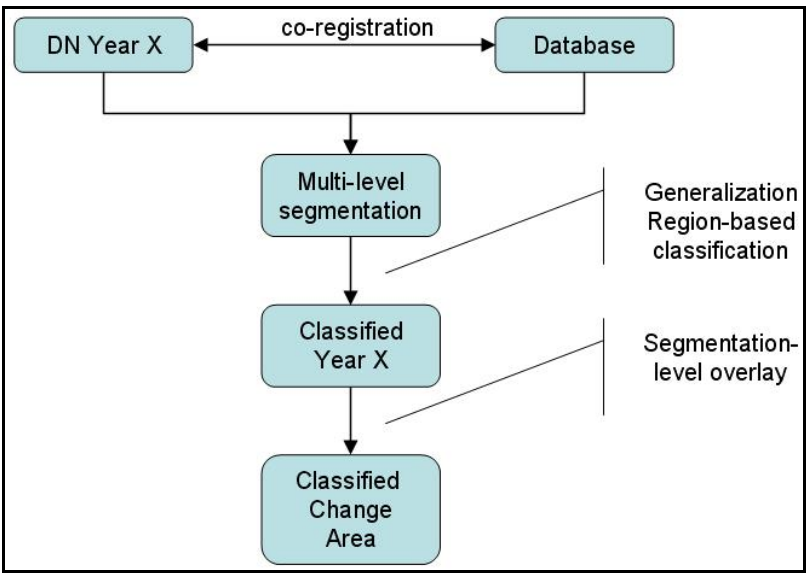

Figure 1. Used change detection method

\subsection{Co-registration}

Of the various requirements of preprocessing for change detection, spatial registration and radiometric calibration are the most important (Lu et al., 2003, Prenzel, 2004, Rogan and Chen, 2004). Due to the used method the radiometric calibration is useless but the importance of accurate spatial registration of multi-temporal imagery and databases is obvious because largely spurious results of change detection will be produced if there is misregistration (Lu et al., 2003, Coppin et al., 2004). The QuickBird image was orthorectified according to the database and with a 10x10 m DTM available on the study zone.

The geometric registration error between the images and the IGN database is expressed in terms of an acceptable total root mean square error (RMSE), which represents a measure of deviation of corrected GCP coordinate values from the original references GCPs used to develop the correction model. The lower RMSE achieved in this process is necessary to reduce the possibility of any false change detections due to misregistration of the co-registered image and database. The table $\mathrm{x}$ presents the RMSE for the panchromatic and XS QuickBird images.

Table 2. RMSE for the QuickBird panchromatic and XS images (expressed in pixel of the orthorectified image)

\begin{tabular}{|c|r|r|r|}
\cline { 2 - 4 } \multicolumn{1}{c|}{} & Nb GCP & X RMSE & Y RMSE \\
\hline Panchromatic & 48 & 0,98 & 1,54 \\
\hline XS & 48 & 0,25 & 0,39 \\
\hline
\end{tabular}

\subsection{Segmentation}

The segmentation technique used in this project will be a bottom up "Region Growing" technique implemented in the eCognition software. The procedure starts at each point in the image with one-pixel regions and in numerous subsequent steps, smaller image regions are merged into bigger ones until a certain heterogeneity value (scale parameter) is reached. The larger the scale parameter, the larger the image regions. This segmentation technique is not very sensitive to the texture (Carleer et al., 2005), very present in VHR data, and makes it possible to segment the image on several levels (multi-level). Each level is defined by a growing scale parameter value and is made up of the merger of the lower level regions. It was shown that in fact a single optimal scale could not accurately represent all classes in a complex scene, due to the contrasting sizes, shapes, and internal variation of the patches for different landcover classes (Raptis et al., 2003, Ju et al, 2005). The multilevel segmentation allows to identify different objects in different segmentation level.

Like said above, the database is used as basis of the image segmentation (Segmentation level 1) and each region of the database is segmented in order to detect the different objects that compose them (Segmentation level2) (Figure 3).
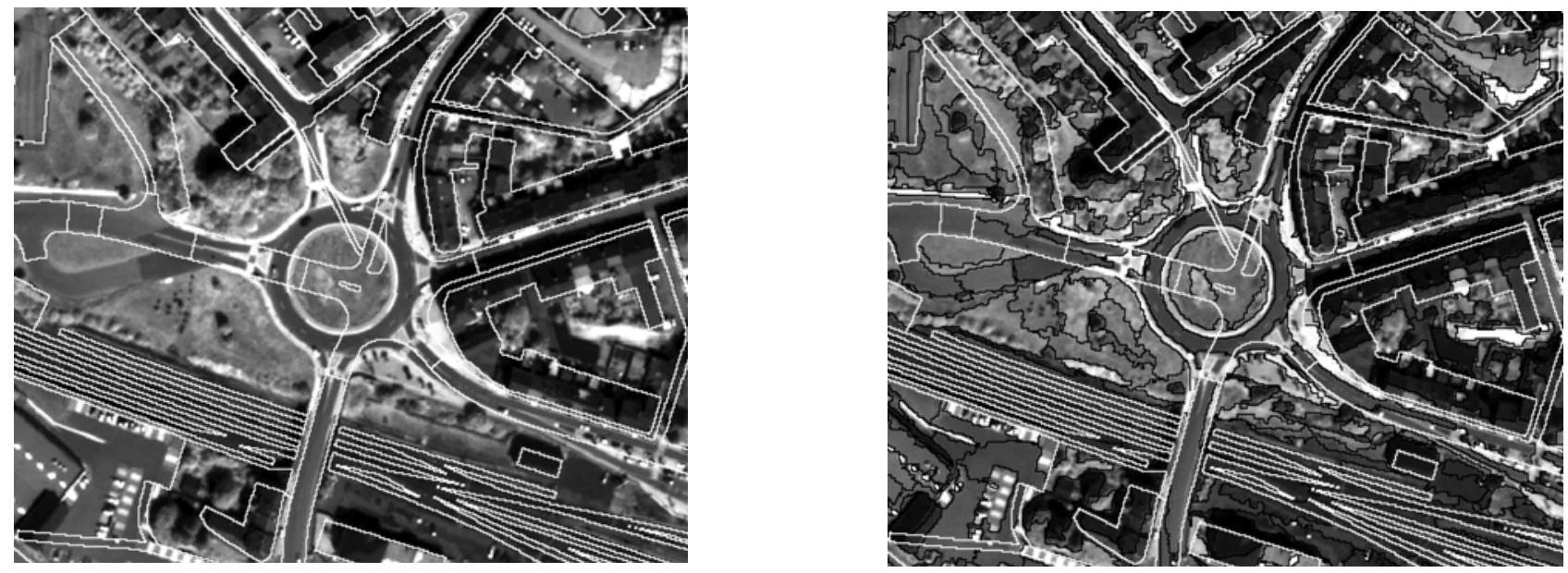

Figure 3.Extract of the orthorectified panchromatic QuickBird image, (A) segmentation level 1 constrained by the vector database and (B) segmentation level 2. 


\subsection{Classification and change detection}

The first segmentation level (constrained by the database) was classified according to the database classes in order to recover the database information. At this step a semantic generalization was carried out. The 93 Land-use/Land-cover classes were generalized in 5 classes: Barren surface, Building, Road, Vegetation and Water. For example, the 22 vegetation classes of the TOP10V-GIS database were gathered together in one Vegetation class.

The second segmentation level was classified according to the same legend but with image features (spectral, textural and morphologic) with the Nearest Neighbor classification method.

The features calculated on the regions can be numerous and they all cannot be used. Using all of them not only makes computer system slow, but compromises the accuracy of the classification (Penaloza and Welch, 1996, Guindon, 2000). A selection of relevant features for each class is then essential to minimize the redundant signatures. In order to find the most suitable features for each class, the public domain program "Multispec" was used. This program was designed for the analysis of multispectral and hyperspectral image data. The most suitable features are found by calculation of class separability based on the Bhattacharyya distance.

The Bhattacharyya distance provides a separability score between each land cover class for a given set of features. This information can be used to identify the features that contribute the largest amount of separation of these classes. The Bhattacharyya distance measure is derived from training areas selected for each class. These training areas were selected by an expert as it is commonly done. Then, the best combination of four features is used in the classification process.

Both segmentation levels were overlaid in order to detect the changes or transitions

\subsection{Change detection evaluation}

Commonly, the change detection is assessed by different kind of matrixes completed by overlaying the change detection results with a reference. From the change/no change error matrix one can calculate how accurately change was distinguished from no change. But in the case of method that distinguish land-cover classes it is not meant for reporting the correctness of class assignment and therefore it does not report the frequency of "true no change, incorrect class" errors, nor does it report the frequency of "true change, incorrect class" errors. If done is also interested in the latter, then one needs the full transition error matrix (van Oort, 2007).

According to the legend, a transition matrix was completed for each one of the 25 transition classes.

From these two matrixes, the change detection accuracy and the transition detection accuracy are calculated.

\section{RESULTS}

The results of the "from-to" change detection are presented in the transition and change/no change matrixes (Table 5 and 6).

The change detection accuracy and the transition detection accuracy can be both calculated from the transition matrix.

The change detection accuracy $=\mathbf{7 2 . 3 \%}$, and

the transition detection accuracy $=\mathbf{7 0 . 3} \%$.

These results are good and show that the changes were distinguished well from no changes, and that the classes are well classified. But the transition matrix highlights other interesting values.
The classification detects seventh as much change than the reference but detects $\mathbf{9 0 . 5 \%}$ of the true changes.

The fact that the classification detects seventh as much change is easily explained. In the images there are a lot of occlusions of the roads, buildings and other surfaces by vegetation (trees) or shadows. Moreover, changes are detected in some regions like garden or crop fields. The gardens and crop fields classes in the database are considered like vegetation but parts of them are not covered by vegetation. There are terraces, paths or car ways and all these parts are detected like changes compared as vegetation. The $70.5 \%$ of change detection is explained in part by the classification errors but also by the class definition outlined above.

Table 5. The "Change" detection matrix

\begin{tabular}{l|l|r|r|}
\multicolumn{3}{c}{} & \multicolumn{2}{c}{ Reference } \\
\cline { 2 - 4 } Classification & & No change & Change \\
\cline { 2 - 4 } & No change & 8853285 & 53638 \\
\cline { 2 - 4 } & Change & 3538499 & 514564 \\
\cline { 2 - 4 } & &
\end{tabular}

Change detection accuracy $=72,3 \%$

Table 6. The “Transition” detection matrix

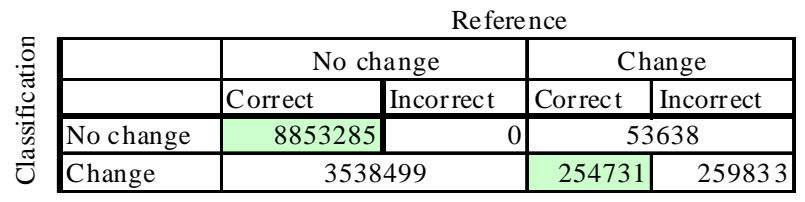

Transition detection accuracy $=70,3 \%$

\section{CONCLUSION}

This study case presents encouraging results in the change detection process integrating region-based classification.

Solution must be found to overcome the class confusion and the occlusion problem. Also, the shadow remains a great problem in the interpretation of VHR satellite data even if some solutions based on shadow interpretation were investigated (Carleer and Wolff, 2006, Dare, 2005).

For the database land-use classes, Steinnocher and Kressler (2006) proposed a solution. They detected the changes between a vector geospatial database and VHR satellite images. The aim of the analysis is to highlight those areas where changes are likely to have taken place. Depending on the number of classes and the aggregation level of the reference data the number of classes can be very different compared to those of the classification. For this reason comparison is carried out on the basis of plausibility as opposed to creating a change/no change map. Depending on which class is present in the reference data and which is in the classification, a segment is either assigned to the change class identical, plausible, questionable or new.

This study highlights the difficulties of the use of VHR satellite images to detect change from a land cover/use database in an automatic way (land use classes, occlusion, shadow ...) but solutions are in progress and the study shows the advantages of the VHR satellite images use instead of aerial images.

\section{REFERENCES}

Aplin, P., Atkinson, P.M., Curran, P.J., 1997, Fine spatial resolution satellite sensors for the next decade, International journal of remote sensing, 18(18), 3873-3881. 
Aplin, P., Atkinson, P.M., Curran, P.J., 1999, Fine spatial resolution simulated satellite sensor imagery for land cover mapping in the United-Kingdom, Remote sensing of environment, 68, 206-216.

Carleer A.P., E. Wolff (2006) Urban land cover multi-level region-based classificationof VHR data by selecting relevant features, International Journal of Remote Sensing, 27(5-6), 1035-1051.

Carleer A.P., O. Debeir, E. Wolff (2005) Assessment of very high spatial resolution satellite image segmentations, Photogrammetric Engineering \& Remote Sensing, 71(11), 1285-1294.

Coppin P., Jonckeere I., Nackaerts K., Muys B., 2004, Digital change detection methods in ecosystem monitoring: a review, International journal of remote sensing, 25(9), 1565-1596.

Cushnie J.L., 1987, The interative effect of spatial resolution and degree of internal variability within land cover types on classification accuracies, International journal of remote sensing, 8(1), 15-29.

Dai X.L., Khorram S., 1999, Remotely sensed change detection based on artificial neural networks, Photogrammetric engineering and remote sensing, 65(10), 1187-1194.

Dare P.M. (2005). "Shadow analisys in high-resolution satellite imagery of urban areas." Photogrammetric engineering and remote sensing, 71(2), 169-177.

De Wit A.J.W., Clevers J.G.P.W., 2004, Efficiency and accuracy of per-field classification for operational crop mapping, International journal of remote sensing, 25(20), 40914112 .

Ferro C.J.S., Warner T.A., 2002, Scale and texture in digital image classification, Photogrammetric engineering and remote sensing, 68(1), 51-63.

Fuller R.M., Smith G.M., Devreux B.J., 2003, The characterisation and measurement of land cover change through remote sensing: problems in operational applications ?, International journal of applied earth observation and geoinformation, 4, 243-253.

Gong P., 1994, Reducing boundary effects in a kernel-based classifier, International journal of remote sensing, 15(5), 11311139.

Guindon, B. (2000). Combining diverse spectral, spatial and contextual attributes in segment-based image classification. ASPRS 2000 Annual Conference, Washington DC.

Haralick, R.M., Shanmugam, K., Dinstein I., 1973, Textural features for image classification, IEEE Transactions on systems, man, and cybernetics, 3(6), 610-621.

Herold, M., Gardner, M.E., Roberts, D.A., 2003, Spectral resolution requirements for mapping urban areas, IEEE Transactions on geoscience and remote sensing, 41(9), 19071919.

Herold, M., Scepan, J., Muller, A., Gunther, S. (2002). Objectoriented mapping and analysis of urban land use/cover using IKONOS data. Proccedings of the 22nd EARSEL Symposium,
Geoinformation for Enropean-Wide Integration, Prague, Czech Republic, Millpress, Rotterdam, The Netherlands.

Irons, J.R., Markam, B.L., Nelson, R.F., Toll, D.L., Williams, D.L., Latty, R.S., Stauffer, M.L., 1985, The effect of spatial resolution on the classification of Thematic Mapper data, International journal of remote sensing, 6(8), 1385-1403.

Jensen, J.R., Cowen, D.C., 1999, Remote Sensing of Urban/Suburban infrastructure and socio-economic attributes, Photogrammetric engineering and remote sensing, 65(5), 611622.

Johnsson K., 1994, Segment-based land-use classification from SPOT satellite data, Photogrammetric engineering and remote sensing, 60(1), 47-53.

Ju, J., Gopal, S., Kolaczyk, E.D., 2005, On the choice of spatial and categorical scale in remote sensing land cover classification, Remote sensing of environment, 96, 62-77.

Key, T., Warner, T.A., McGraw, J.B., Fajvan, M.A., 2001, A comparison of multispectral and multitemporal information in high spatial resolution imagery for classification of individual tree species in a temperate hardwood forest, Remote sensing of environment, 75, 100 - 112.

Knudsen T., Olsen B., 2003, Automated change detection for updates of digital map databases, Photogrammetric engineering and remote sensing, 69(11), 1289-1296.

Lillesand, Kiefer (1994), Remote sensing and image interpretation. Wiley and Sons, Inc., New York, pp. 413.

Loveland TR, Sohl TL, Stehman SV, Gallant A.L., Sayler K.L., Napton D.E., 2002, A strategy for estimating the rates of recent United States land-cover changes, Photogrammetric engineering and remote sensing, 68 (10): 1091-1099.

Lu D., Mausel P., Brondizio E., Moran E., 2003, Change detection techniques, International journal of remote sensing, 25(12), 2365-2407.

Marçal A.R.S., Borges J.S., Gomes J.A., Pinto Da Costa J.F., 2005, Land cover update by supervised classification of segmented ASTER images, International journal of remote sensing, 26(7), 1347-1362.

Marceau D.J., Howarth P.J., Dubois J-M.M., Gratton D.J., 1990, Evaluation of the grey-level co-occurence matrix method for land cover classification using SPOT imagery., IEEE Transactions on geoscience and remote sensing, 28(4), 513-519.

Petit C.C., Lambin E.F., 2001, Integration of multi-source remote sensing data for land over change detecion, International journal of geographical information science, 15(8), 785-803.

Prenzel B., 2004, Remote sensing-based quantification of landcover and land-use change for planning, Progress in planning, 61(4), 281-299.

Raptis, V.S., Vaughan, R.A., Wright, G.G., 2003, The effect of scalling on land cover classification from satellite data, Computers and geosciences, 29, 705-714.

Rogan J., Chen D.M., 2004, Remote sensing technology for mapping and monitoring land-cover and land-use change, Progress in planning, 61(4), 301-325. 
Ryherd S., Woodcock C., 1996, Combining spectral and texture data in the segmentation of remotely sensed images, Photogrammetric engineering and remote sensing, 62(2), 181194.

Segl K., Kaufmann H., 2001, Detection of small objects from high-resolution panchromatic satellite imagery based on supervised image segmentation, IEEE Transactions on geoscience and remote sensing, 39(9), 2080-2083.

Shackelford A.K., C.H. Davis, 2003, A hierarchical fuzzy classification approach for high-resolution multispectral data over urban areas, IEEE Transactions on geoscience and remote sensing, 41(9), 1920-1932.

Smith G.M., Fuller R.M., 2001, An integrated approach to land cover classification: an example in the Island of Jersey, International journal of remote sensing, 22(16), 3123-3142. $\mu$

Steinnocher K., Kressler F., 2006, EuroSDR Official Publication no. 50, EuroSDR Project - Change Detection, 112182.

Thomas, N., Hendrix, C., Congalton, R.G., 2003, A comparison of urban mapping methods using high-resolution digital imagery, Photogrammetric engineering and remote sensing, 69(9), 963-972.

Treitz P., J. Rogan, 2004, Remote sensing for mapping and monitoring land-cover and land-use change - an introduction, Progress in planning, 61(4), 269-279.

van Oort, P.A.J., 2007, Interpreting the change detection error matrix, Remote Sensing of Environment, 108, 1-8.

Walter, V., 2004, Object-based classification of remote sensing data for change detection, ISPRS Journal of photogrammetry and remote sensing, 58, 225-238.

Weis, M., Müller, S., Liedtke, C.-E., Palh, M., 2005, A framework for a GIS and imagery data fusion in support of cartographic updating, Information fusion, 6, 311-317.

Woodcock, C.E., Strahler, A.H., 1987, The factor of scale in remote sensing, Remote sensing of environment, 21, 311-332.

\section{AKNOWLEDGEMENTS}

The image data were funded by the European Commission and made available by the JRC - IPSC - GeoCAP unit through the Ministry of Agriculture (Walloon Region, Belgium).

This research is carried out in the framework of the Belgian ORFEO accompaniment program and funded by the Belgian Science Policy (BelSPo) - contract n ${ }^{\circ} \mathrm{OR} / 11 / 04$. 